\title{
Genetic variation in DLG5 is associated with inflammatory bowel disease
}

\author{
Monika Stoll $^{1,7}$, Brit Corneliussen ${ }^{2}$, Christine M Costello ${ }^{1}$, Georg H Waetzig ${ }^{3}$, Bjorn Mellgard ${ }^{2}$, W Andreas Koch ${ }^{1}$, \\ Philip Rosenstiel ${ }^{1}$, Mario Albrecht ${ }^{4}$, Peter J P Croucher ${ }^{1}$, Dirk Seegert ${ }^{3}$, Susanna Nikolaus ${ }^{1}$, Jochen Hampe ${ }^{1,5}$, \\ Thomas Lengauer ${ }^{4}$, Stefan Pierrou ${ }^{2}$, Ulrich R Foelsch ${ }^{1}$, Christopher G Mathew ${ }^{6}$, Maria Lagerstrom-Fermer ${ }^{2} \&$ \\ Stefan Schreiber ${ }^{1,5}$
}

Crohn disease and ulcerative colitis are two subphenotypes of inflammatory bowel disease (IBD), a complex disorder resulting from gene-environment interaction. We refined our previously defined linkage region for IBD on chromosome 10q23 and used positional cloning to identify genetic variants in DLG5 associated with IBD. DLG5 encodes a scaffolding protein involved in the maintenance of epithelial integrity. We identified two distinct haplotypes with a replicable distortion in transmission $(P=$ 0.000023 and $P=0.004$ for association with IBD, $P=0.00012$ and $\boldsymbol{P}=0.04$ for association with (rohn disease). One of the riskassociated DLG5 haplotypes is distinguished from the common haplotype by a nonsynonymous single-nucleotide polymorphism $113 G \rightarrow A$, resulting in the amino acid substitution $R 30 Q$ in the DUF622 domain of DLG5. This mutation probably impedes scaffolding of DLG5. We stratified the study sample according to the presence of risk-associated CARD15 variants to study potential gene-gene interaction. We found a significant difference in association of the 113A DLG5 variant with Crohn disease in affected individuals carrying the risk-associated CARD15 alleles versus those carrying non-risk-associated CARD15 alleles. This is suggestive of a complex pattern of genegene interaction between DLG5 and CARD15, reflecting the complex nature of polygenic diseases. Further functional studies will evaluate the biological significance of DLG5 variants.

Figure 1 Genetic variants of DLG5 are associated with IBD. The experimental steps from linkage mapping on the long arm of chromosome 10 to identification of genetic variants in DLG5 associated with IBD, Crohn disease (CD) and ulcerative colitis (UC) are shown. Annotation of the most important microsatellite and SNP markers that led to the identification of $D L G 5$ as a susceptibility gene for IBD and Crohn disease is also shown. The markers with strongest association with the IBD phenotype are indicated with bold vertical tick marks. The corresponding association results are presented in detail in Tables $\mathbf{1}$ and $\mathbf{2}$ and Figure $\mathbf{3}$.
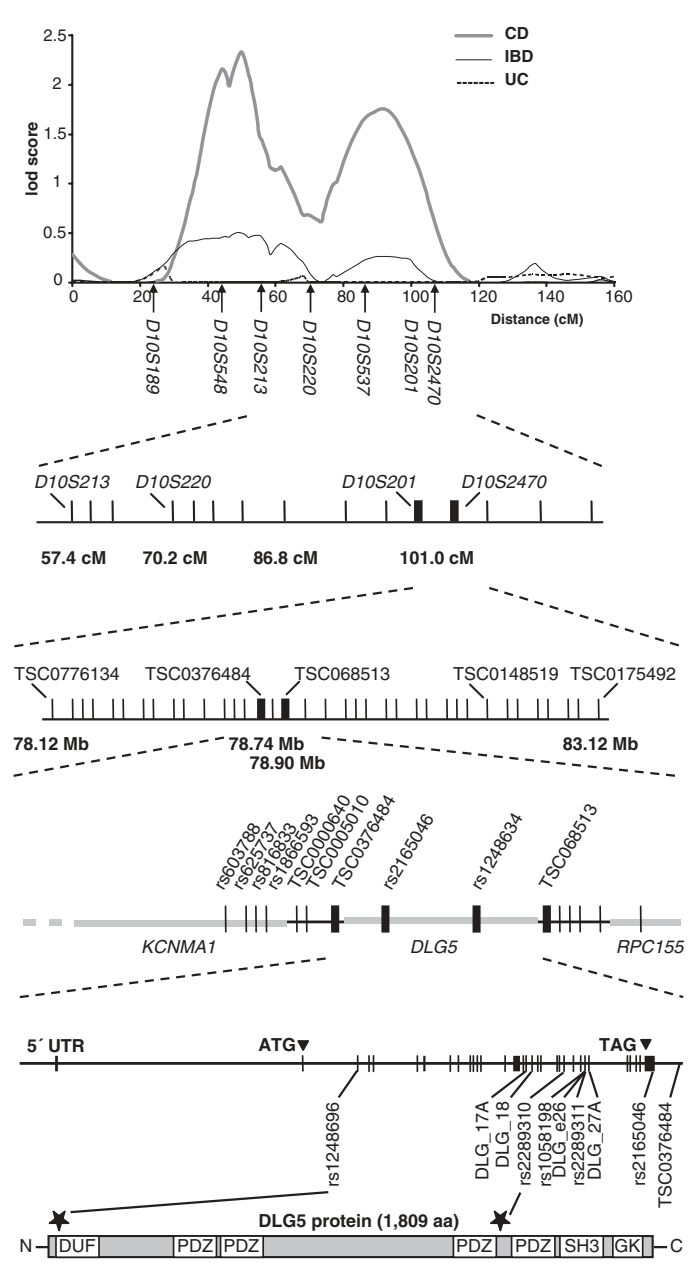

${ }^{1}$ First Department of Medicine, University Hospital Schleswig-Holstein, Schittenhelmstr. 12, 24105 Kiel, Germany. ${ }^{2}$ AstraZeneca, Experimental Medicine and Department of Molecular Sciences, R\&D Molndal, Sweden. ${ }^{3}$ Conaris Research Institute AG, Kiel, Germany. ${ }^{4}$ Max-Planck-Institute for Informatics, Saarbrücken, Germany. ${ }^{5}$ Institute for Clinical Molecular Biology, Christian-Albrechts-University, Kiel, Germany. ${ }^{6}$ Guy's, King's and St. Thomas' School of Medicine, London, UK. ${ }^{7}$ Present address: Genetic Epidemiology of Vascular Disorders, Institute for Arteriosclerosis Research, Muenster, Germany. Correspondence should be addressed to S.S. (s.schreiber@mucosa.de).

Published online 11 April 2004; doi:10.1038/ng1345 
IBD is a spectrum of chronic relapsing inflammatory disorders affecting the gastrointestinal tract that can be classified into Crohn disease and ulcerative colitis. The identification of CARD15 (refs.1-3) and several loci associated with susceptibility to IBD in independent linkage studies ${ }^{4}$ documents the polygenic etiology of IBD. We previously identified a locus in the pericentromeric region of chromosome 10 that was associated with susceptibility to IBD in a genome-wide linkage scan involving 282 families of European descent ${ }^{5}$. Fine mapping at an average distance of $5 \mathrm{cM}$ using an additional 11 microsatellite markers in an extended linkage cohort (111 additional families including 422 affected sibling pairs) confirmed initial linkage findings and identified a two-peak linkage curve extending from D10S547 to D10S192 (multipoint lod score = 2.07, $P=0.0033$ at D10S548) and a second peak at D10S201 (multipoint lod score $=1.6$ ) for associated with Crohn disease (Fig. 1). We used a hierarchical linkage disequilibrium (LD) study to search for the causal variant(s) in the 40-cM interval (Fig. 1). Transmission disequilibrium testing (TDT) of trios randomly drawn from each family showed a significant single-point association with Crohn disease at D10S201 $(P<$ $0.01)$, located in the second linkage peak on 10q22-10q23. We finely mapped the underlying 5-Mb region at an average distance of 75-120 kb using 37 single-nucleotide polymorphisms (SNPs) selected from the TSC allele frequency project in 457 independent trios with IBD (Supplementary Table 1 online). The marker TSC0376484 (rs1344966) in this panel was significantly associated with Crohn disease $\left(\chi^{2}=9.00\right.$, $P=0.002$ ) and more strongly with IBD (Crohn disease and ulcerative colitis, $\chi^{2}=11.65, P=0.0006$; Fig. 1).
TSC0376484 is located near two genes of possible (patho)physiological relevance to chronic intestinal inflammation: KCNMA1, encoding a potassium-gated calcium channel ${ }^{6}$, and DLG5, a member of the membrane-associated guanylate kinase gene family, which is important in the maintenance of epithelial cell integrity ${ }^{7}$. To genetically narrow the association signal to one single candidate, we used LD mapping and genotyped selected publicly available SNPs from each gene in the 457 trios with IBD. The association signal was confined to $D L G 5$, and none of the markers in KCNMA1 was significantly associated with IBD (Supplementary Table 2 online).

We sequenced coding exons 2-32 and the exon-intron boundaries of DLG5 in 47 individuals with IBD and identified or verified 33 SNPs (Supplementary Tables 3 and 4 online). We then tested all these SNPs for disease association. Association with the IBD phenotype was strongest (Table 1), with 18 markers in DLG5 showing significant association. Separate analysis of the Crohn disease and ulcerative colitis subgroups showed a strong association in the Crohn disease subgroup, which is in accordance with the original linkage observation ${ }^{5}$. The weaker signal in the ulcerative colitis subgroup may be due to reduced power in a small sample size. Because the combined group had the strongest association, we suggest that the signal in DLG5 reflects a factor associated with general susceptibility to IBD rather than to Crohn disease only.

Pairwise LD measures $\left(\mathrm{D}^{\prime}\right)$ indicated strong LD across the entire gene, defining a single haplotype block of $\sim 85 \mathrm{~kb}$ and $\mathrm{D}^{\prime}$ values $>0.8$, except at TSC0000361 (located on the neighboring LD segment). We

Table 1 Summary of TDT results in German trios for single-point association with IBD, Crohn disease and ulcerative colitis

\begin{tabular}{|c|c|c|c|c|c|c|c|c|c|}
\hline \multirow[b]{2}{*}{ Assay name } & \multicolumn{3}{|c|}{$\mathrm{IBD}(n=457)$} & \multicolumn{3}{|c|}{ Crohn disease $(n=302)$} & \multicolumn{3}{|c|}{ Ulcerative colitis $(n=155)$} \\
\hline & $\mathrm{T}: \mathrm{U}$ & $x^{2}$ & $P$ & $\mathrm{~T}: \mathrm{U}$ & $\chi^{2}$ & $P$ & $\mathrm{~T}: \mathrm{U}$ & $\chi^{2}$ & $P$ \\
\hline TSC0000361 & $24: 15$ & 2.077 & 0.1495 & $13: 12$ & 0.04 & 0.8415 & $11: 03$ & 4.571 & 0.0325 \\
\hline rs1248696 & $92: 64$ & 5.026 & 0.0250 & 60:42 & 3.176 & 0.0747 & $32: 22$ & 1.852 & 0.1736 \\
\hline rs1248680 & $151: 191$ & 4.678 & 0.0306 & $102: 127$ & 2.729 & 0.0985 & $64: 49$ & 1.991 & 0.1582 \\
\hline rs1248677 & $203: 180$ & 1.381 & 0.2399 & $138: 123$ & 0.862 & 0.3532 & $57: 65$ & 0.525 & 0.4687 \\
\hline rs1248670 & $172: 159$ & 0.511 & 0.4747 & $117: 109$ & 0.283 & 0.5947 & $50: 55$ & 0.238 & 0.6257 \\
\hline rs1270912 & $201: 182$ & 0.943 & 0.3315 & $134: 124$ & 0.388 & 0.5334 & $58: 67$ & 0.648 & 0.4208 \\
\hline DLG5_e13 & $154: 196$ & 5.4 & 0.0248 & $105: 128$ & 2.24 & 0.1319 & $68: 49$ & 3.085 & 0.0790 \\
\hline rs2289308 & $230: 171$ & 8.681 & 0.0032 & $155: 115$ & 5.926 & 0.0149 & $75: 56$ & 2.756 & 0.0969 \\
\hline rs1248634 & $150: 196$ & 6.116 & 0.0134 & $104: 130$ & 2.889 & 0.0892 & $66: 46$ & 3.571 & 0.0588 \\
\hline DLG5_e17 & $152: 192$ & 4.651 & 0.0310 & $104: 128$ & 2.483 & 0.1151 & $64: 48$ & 2.286 & 0.1305 \\
\hline DLG5_e18 & $236: 175$ & 9.054 & 0.0026 & $158: 119$ & 5.491 & 0.0191 & $78: 56$ & 3.612 & 0.0574 \\
\hline rs2289310 & $40: 24$ & 4.0 & 0.0455 & $24: 17$ & 1.195 & 0.2743 & $07: 16$ & 3.522 & 0.0606 \\
\hline rs1261990 & $147: 196$ & 7.0 & 0.0082 & $100: 131$ & 4.16 & 0.0414 & $65: 47$ & 2.893 & 0.0890 \\
\hline DLG5_e25 & $23: 15$ & 1.684 & 0.1944 & 13:11 & 0.167 & 0.6828 & $04: 10$ & 2.571 & 0.1088 \\
\hline rs1058198 & $228: 166$ & 9.756 & 0.0018 & $151: 111$ & 6.107 & 0.0135 & $77: 55$ & 3.667 & 0.0555 \\
\hline DLG5_e26 & $225: 162$ & 10.256 & 0.0014 & $151: 108$ & 7.139 & 0.0075 & $74: 54$ & 3.125 & 0.0771 \\
\hline rs2289311 & $231: 171$ & 8.955 & 0.0028 & $154: 114$ & 5.97 & 0.0146 & $77: 57$ & 2.985 & 0.0840 \\
\hline DLG5_e27 & $235: 175$ & 8.72 & 0.0030 & $157: 118$ & 5.531 & 0.0187 & $78: 57$ & 3.267 & 0.0707 \\
\hline rs2241833 & $152: 196$ & 5.563 & 0.0183 & $103: 132$ & 3.579 & 0.0585 & $64: 49$ & 1.991 & 0.1582 \\
\hline rs2579150 & $146: 193$ & 6.516 & 0.0107 & $98: 130$ & 4.491 & 0.0341 & $63: 48$ & 2.027 & 0.1545 \\
\hline rs2812425 & $201: 186$ & 0.581 & 0.4459 & $133: 129$ & 0.061 & 0.8049 & $57: 68$ & 0.968 & 0.3252 \\
\hline rs1058202 & $176: 161$ & 0.668 & 0.4137 & $118: 107$ & 0.538 & 0.4633 & $54: 58$ & 0.143 & 0.7053 \\
\hline rs1058203 & $155: 196$ & 4.789 & 0.0286 & $104: 130$ & 2.889 & 0.0892 & $66: 51$ & 1.923 & 0.1655 \\
\hline rs2165046 & $241: 176$ & 10.132 & 0.0015 & $159: 120$ & 5.452 & 0.0195 & $82: 56$ & 4.899 & 0.0269 \\
\hline rs2165047 & $143: 192$ & 7.167 & 0.0074 & $96: 129$ & 4.84 & 0.0278 & $63: 47$ & 2.327 & 0.1271 \\
\hline rs2579151 & $148: 189$ & 4.988 & 0.0255 & $101: 130$ & 3.641 & 0.0564 & $59: 47$ & 1.358 & 0.2439 \\
\hline TSC0376484 & $177: 123$ & 9.72 & 0.0018 & 118:87 & 4.688 & 0.0304 & $59: 36$ & 5.568 & 0.0183 \\
\hline
\end{tabular}

Single-point TDT association for SNP markers located in DLG5 and flanking regions. Association is shown for IBD (Crohn disease and ulcerative colitis combined) and the subphenotypes Crohn disease and ulcerative colitis separately. T:U, transmitted versus untransmitted allele. The association is most pronounced in the IBD group and the Crohn disease subgroup, whereas only few markers show significance or a trend of association with ulcerative colitis, which is in accord with the original linkage finding 5 . 
observed a sharp decline in LD at the boundaries of the haplotype block, differentiating DLG5 from the neighboring genes KCNMA1 and RPC155 (Fig. 2). Analysis of the extended DLG5 haplotype identified four common haplotypes (Fig. 3), with haplotype A tagged by eight SNPs (haplotype-tagging SNPs or htSNPs) of equivalent genetic information content. Haplotype A was significantly undertransmitted to individuals with IBD and Crohn disease, whereas haplotype D, uniquely tagged by the coding variant 113A, was significantly overtransmitted to individuals with both $\operatorname{IBD}\left(\chi^{2}=8.08, P=0.004\right)$ and Crohn disease $\left(\chi^{2}=4.15, P=0.04\right.$; Fig. 3$)$.

To corroborate our initial association finding, we genotyped the DLG5 htSNPs in an independent sample consisting of trios with IBD who had not yet been analyzed ( $n=485$; Supplementary Table 1 online). The htSNP DLG5_e26 in haplotype A was undertransmitted to the individuals with IBD (transmitted:untransmitted (T:U) ratio of 165:214), replicating the observed association $(P=$ 0.006 in a one-tailed test), and rs $1058198 \mathrm{had}$ a T:U ratio of $196: 237(P=0.024) .113 \mathrm{~A}$ was overtransmitted in both IBD (T:U 90:73, $P=0.09$ ) and Crohn disease (T:U 58:43, $P=0.065$ ) but the distortion was not statistically significant. This can be explained by the smaller proportion of trios with Crohn disease in our replication sample and the reduced power in replication situations. We therefore tested the associated markers in a second independent sample (538 Crohn disease cases and 548 controls) using the case-control study design to estimate the attributable risk in a diverse population of European descent. The 113A variant was significantly associated with the IBD phenotype $(P=0.001$, odds ratio $(\mathrm{OR})=1.62)$ as was $\mathrm{rs} 2289310$ $(4136 \mathrm{C} \rightarrow \mathrm{A}$, resulting in the amino acid substitution $\mathrm{P} 1371 \mathrm{Q} ; P=0.01, \mathrm{OR}=1.51$ ). DLG5_e26, tagging haplotype A, was significantly associated with IBD (Table 2), providing a second independent replication of association. The combined $P$ values for the repeated, independent associations with IBD $(n=2)$ were 0.029 for $113 \mathrm{~A}$ and 0.0007 for DLG5_e26, and those for the repeated, independent associations with Crohn disease $(n=$ 3 ) were $P=0.001$ for $113 \mathrm{~A}$ and $P=0.0004$ for DLG5_e26.

Because the $4136 \mathrm{~A}$ risk allele is not included on the common haplotypes carrying $113 \mathrm{~A}$, but instead on a rare haplotype (frequency $<1 \%$ ), we calculated the global differences in genotype combinations for 113A and $4136 \mathrm{~A}$ to estimate the risk for homozygosity or compound heterozygosity. This analysis identified a significant difference in genotype frequencies (global $\chi^{2}=13.61, P=0.0029$ ) in individuals with IBD compared with healthy controls. The OR was 1.74 (95\% confidence interval $=1.31-2.32)$ for individuals carrying genomic segments.

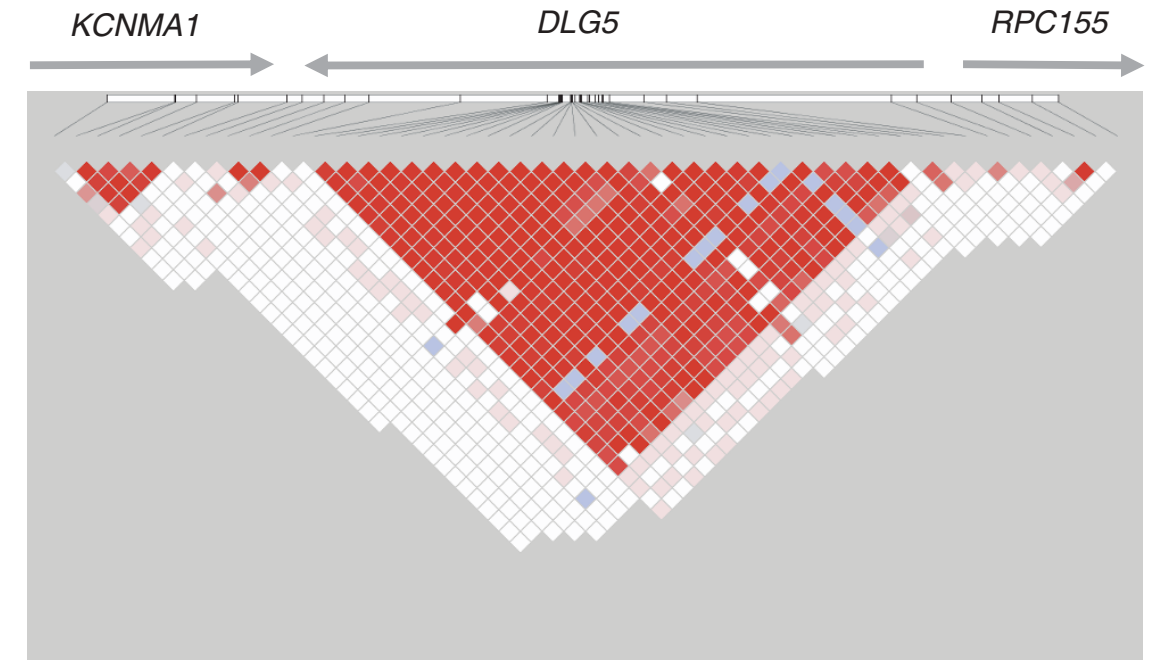

Figure 2 LD across DLG5 and flanking genomic region. D' values for pairwise LD between each marker (red $=D^{\prime}>0.8$ ) are represented. Top of figure shows spacing of markers across the genomic region. All markers in DLG5 (large red block) are in strong LD. LD drops off sharply (white blocks) at the boundaries of DLG5, indicating that the neighboring genes are located on different (independent)

at least two risk alleles (113A and/or 4136A), suggesting that the overall clinical impact of rare single coding mutations such as $4136 \mathrm{~A}$ on the IBD phenotype is limited. Our disease model that links $113 \mathrm{~A}$ and $4136 \mathrm{~A}$ to the positional signal detected in DLG5 is further supported by the identification of rare, coding, 'private' variants (resulting in the amino acid substitutions S121G, E514Q, R957H and P979L; frequency $<0.5 \%$ ) through systematic sequence analysis in 47 individuals.

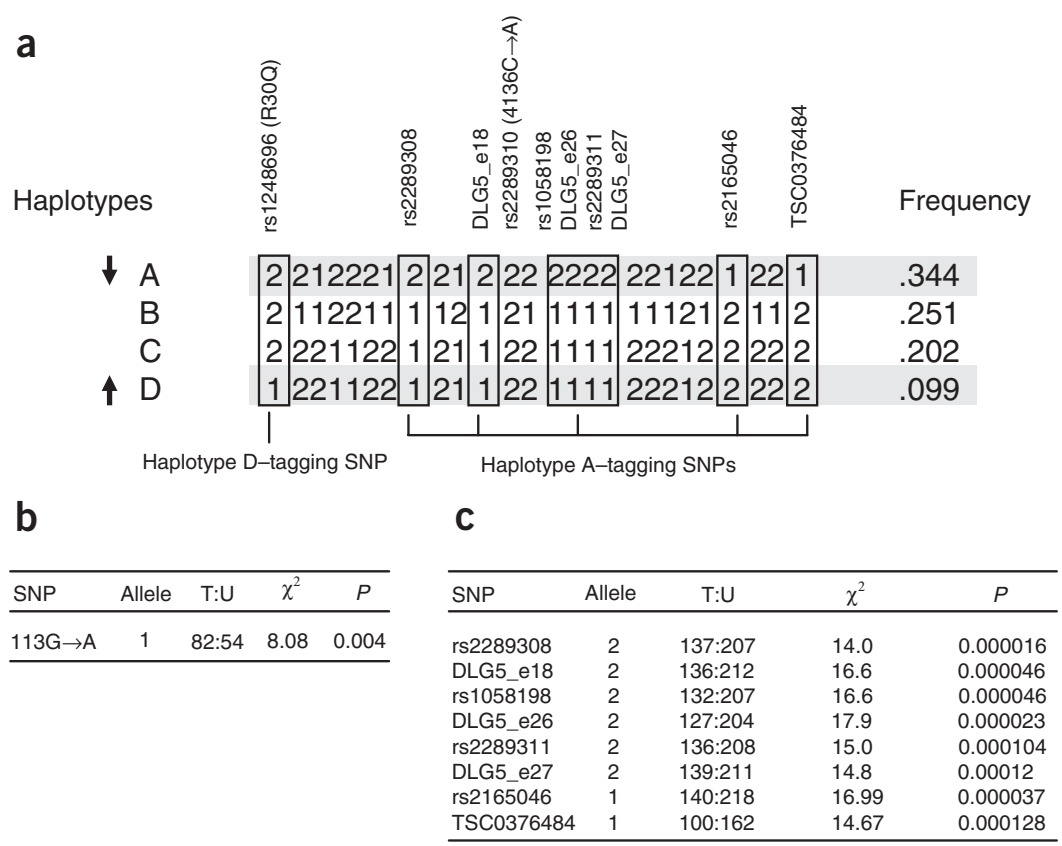

Figure 3 Multilocus haplotype results. (a) The four most common haplotypes for DLG5. The boxed alleles refer to the htSNPs. Haplotype D carries the risk-conferring htSNP rs1248696 $(113 \mathrm{G} \rightarrow \mathrm{A})$ and is significantly overtransmitted in trios with IBD and Crohn disease (TDT results shown in b). Haplotype $A$ is tagged by eight htSNPs that carry equivalent genetic information and is significantly undertransmitted in trios with IBD and Crohn disease (TDT results in shown in $\mathbf{c}$ ). 
Table 2 Association of DLG5 SNPs with IBD in an independent case-control sample

\begin{tabular}{|c|c|c|c|c|c|}
\hline $\begin{array}{l}\text { SNP marker } \\
\text { and genotypes }\end{array}$ & Cases & Controls & $\chi^{2}$ & $P$ & $\begin{array}{c}\text { OR } \\
\text { (95\% confidence interval) }\end{array}$ \\
\hline \multicolumn{6}{|l|}{$113 \mathrm{G} \rightarrow \mathrm{A}$} \\
\hline $1-1$ & 7 & 5 & 10.18 & 0.001 & $1.62(1.2-2.2)^{a}$ \\
\hline $1-2$ & 125 & 83 & & & \\
\hline $2-2$ & 393 & 427 & & & \\
\hline \multicolumn{6}{|l|}{$4136 C \rightarrow A$} \\
\hline $1-1$ & 5 & 0 & 6.29 & 0.01 & $1.51(0.92-2.57)^{\mathrm{a}}$ \\
\hline $1-2$ & 34 & 26 & & & \\
\hline $2-2$ & 486 & 490 & & & \\
\hline \multicolumn{6}{|l|}{ DLG5_e26 } \\
\hline $1-1$ & 243 & 207 & 5.73 & 0.01 & $1.35(1.05-1.72)^{\mathrm{b}, \mathrm{c}}$ \\
\hline $1-2$ & 209 & 249 & & & \\
\hline $2-2$ & 62 & 63 & & & \\
\hline
\end{tabular}

a95\% confidence interval for genotypes 1-1 and 1-2 combined (allele 1 as risk allele). b95\% confidence interval for genotypes 1-2 and 2-2 combined (allele 1 as risk allele). Inverse OR for undertransmitted allele 2 was 0.74 .

We were interested in the hypothetical impact of the associated variants, R30Q and P1371Q, on the function of the DLG5 protein. DLG5 has been implicated in regulating cell growth and maintaining cell shape and polarity ${ }^{8}$. A recent study ${ }^{9}$ suggested an epithelial function for DLG5 as a binding partner of vinexin at sites of cell-cell contact, and our preliminary results on expression of DLG5 mRNA in a variety of tissues confirm the presence of the transcript in the colon, the intestine and isolated intestinal epithelial cells (Supplementary Fig. 1 online). It is therefore conceivable that DLG5 has a role in maintaining epithelial structure and that genetic variants in DLG5 interfere with epithelial barrier function in the colon.

DLG5 contains one DUF622 domain, four PDZ domains and one SH3 domain followed by one guanylate kinase domain (Fig. 1) $)^{10,11}$. All these domains are assumed to be involved in protein-protein interactions, supporting the idea that DLG5 is a multifunctional adapter and scaffold protein. We carried out in silico analysis of the potential structural and functional implications of the variants R30Q and P1371Q (Supplementary Fig. 1 online). The results of this analysis suggested that both variants probably impair the scaffolding functions of DLG5 (Supplementary Methods and Supplementary Fig. 1 online).
Finally, we examined potential locus-locus interactions between variants of DLG5 and variants of CARD15, the first susceptibility gene identified for Crohn disease ${ }^{1-3}$. Genetic susceptibility to Crohn disease is mainly conferred by three polymorphisms that induce structural changes in the leucine-rich repeats of CARD15 (R702W, G908R and 3020insC). The allele frequencies of these SNPs range from $\sim 4 \%$ to $14 \%$ in the cohorts with Crohn disease examined to date, and $\sim 30-40 \%$ of individuals with Crohn disease are heterozygous for at least one of the variants, compared with $\sim 10 \%$ of control subjects ${ }^{1-3}$. We examined interactions between DLG5 and CARD15 by stratifying trios in two groups according to the genotype of the affected child. In trios with IBD and Crohn disease, haplotype A (represented by DLG5_e26) was undertransmitted in the groups carrying both the risk-associated and non-risk-associated variants of CARD15 (Table 3), which suggests that haplotype A reflects genetic variation that acts independently of CARD15 variants. In trios with Crohn disease, we observed significantly greater transmission of $113 \mathrm{~A}$ in individuals carrying the risk-associated versus non-risk-associated variants of CARD15 (Table 3 ). This suggests that the $113 \mathrm{~A}$ variant is of particular relevance in individuals with Crohn disease and, further, that an interaction may exist between the risk-associated haplotype of DLG5 and the risk-associated variants of CARD15.

We found replicated association between genetic variations in DLG5 and the risk of developing IBD. The risk-associated DLG5 haplotype D is uniquely distinguished by the $113 \mathrm{~A}$ variant and is suggested to be causative, as are rare, private SNPs. The conferred risk is moderate, which is in agreement with a polygenic disease model. Genetic interaction studies suggest interactions between CARD15 variants and $113 \mathrm{~A}$ in $D L G 5$, but these studies are not yet conclusive and will require large, consolidated efforts by several groups to achieve appropriate statistical power. Future studies in diverse and very large samples are needed to evaluate the population relevance of variants in DLG5 in this chromosomal region. Functional studies need to define the molecular properties of DLG5 variants and their influence on the clinical presentation of IBD.

Table 3 Association of DLG5 haplotypes and interaction with risk-associated CARD15 alleles

\begin{tabular}{|c|c|c|c|c|c|c|}
\hline Haplotype & Phenotype & CARD15 allele ${ }^{a}$ & TDT $(T: U)^{b}$ & $\chi^{2}$ & $P$ & Group comparison $^{c}$ \\
\hline \multicolumn{7}{|c|}{ Haplotype A (DLGe_26 allele 2) } \\
\hline & $\mathrm{IBD}$ & & $162: 225$ & 10.25 & 0.001 & \\
\hline & & Risk-associated & $58: 87$ & 5.8 & 0.01 & \\
\hline & & Non-risk-associated & 97:121 & 2.64 & 0.1 & NS \\
\hline & Crohn disease & & $108: 151$ & 7.13 & 0.007 & \\
\hline & & Risk-associated & $46: 73$ & 6.12 & 0.01 & \\
\hline & & Non-risk-associated & $56: 69$ & 1.35 & 0.24 & NS \\
\hline \multirow[t]{6}{*}{ Haplotype D $(113 G \rightarrow A)$} & IBD & & $92: 64$ & 5.026 & 0.02 & \\
\hline & & Risk-associated & $36: 17$ & 6.81 & 0.009 & \\
\hline & & Non-risk-associated & $51: 43$ & 0.68 & 0.409 & NS \\
\hline & Crohn disease & & $60: 42$ & 3.17 & 0.07 & \\
\hline & & Risk-associated & $30: 12$ & 7.71 & 0.005 & \\
\hline & & Non-risk-associated & $28: 28$ & 0.00 & 1.00 & $P<0.05$ \\
\hline
\end{tabular}

a'Risk-associated' indicates at least one of the mutated CARD15 alleles of SNP8, SNP12 or SNP13. 'Non-risk-associated' indicates wild-type alleles for all three variants. 'PTDT analysis of the DLG5 htSNP DLG5_e26 for allele 2 denoting undertransmitted haplotype A, and 113G $\rightarrow$ A tagging overtransmitted haplotype D (T, number of transmitted chromosomes; U, number of untransmitted chromosomes). This htSNP was genotyped in 457 trios with IBD including 302 trios with Crohn disease and 155 trios with ulcerative colitis. Trios were stratified into groups on the basis of CARD15 genotype and IBD phenotype of the affected child in each trio. ${ }^{c}$ No statistical difference (NS) in the T:U ratios (strength of association) of haplotype A after stratification into groups carrying risk-associated and non-risk-associated CARD15 variants for haplotype A. The statistical difference for haplotype $D$ in the Crohn disease subgroup was determined by a $2-x-2$ contingency table and permutation testing. 


\section{METHODS}

Study samples. Individuals with IBD were recruited by the clinical group through the Charite University Hospital (Berlin, Germany) and at the Department of Internal Medicine I, University Hospital Kiel, Germany. Diagnosis of IBD and subsequent classification into Crohn disease or ulcerative colitis was determined by standard diagnostic criteria ${ }^{12,13}$ and has been described previously ${ }^{3,5,13}$. All individuals were of European descent. We carried out LD mapping in trios consisting of father, mother and child affected with IBD, in which one parent or neither parent was affected with Crohn disease or ulcerative colitis. These trios were identified for LD mapping and have been described $^{3}$. For our confirmatory cohort, we extracted trios randomly from the multicase families used in our previous linkage studies ${ }^{5}$ and supplemented this group with 92 additional trios recruited for this purpose. For case-control association, we compared 538 additional, independent individuals with IBD (singletons) with age- and sex-matched volunteers from the Kiel University blood donation program. All study participants gave informed, written consent. The recruitment protocols and study procedures were approved by the ethics committees of the Charité University Hospital, Berlin, Germany, and the SchleswigHolstein University Hospital, Campus Kiel, Germany, respectively.

Microsatellite typing. In the first stage of microsatellite LD mapping, we genotyped 11 microsatellite markers (D10S547, D10S548, D10S211, D10S611, D10S213, D10S1780, D10S220, D10S1790, D10S609, D10S201 and D10S2470) in 393 families with IBD (422 affected sibling pairs).

Information on primer sequence, allele size range, suggested amplification conditions and genetic position can be obtained from the Genethon and Marshfield databases (see URLs). Genotypes were generated at the University of California Los Angeles using PCR and fluorescence-labeled primers on an ABI 377 sequencer.

SNP discovery in DLG5. To identify all crucial SNPs in the coding sequence of $D L G 5$, as well as exon-intron boundaries and the promoter region, we sequenced 47 individuals with IBD using an ABI 3700 automated sequencer as previously described ${ }^{3}$. The primers and probes for 33 SNPs discovered or verified by resequencing, and the sequences of the new SNPs, are given in Supplementary Tables 3 and 4 online.

SNP genotyping. We selected SNP markers for the initial fine mapping experiment based on information available from the public databases. For analysis of $D L G 5$, we used SNPs generated or verified in-house. We generated SNP genotypes using the TaqMan allelic discrimination method as previously described ${ }^{3}$. Taqman assays were from ABI.

Statistical analysis. We tested each marker for Hardy-Weinberg equilibrium in the control populations using a $\chi^{2}$ test and then carried out genetic analyses at several levels. To confirm the association with Crohn disease, we first subjected each marker to single-locus tests for linkage and transmission disequilibrium testing (TDT) analysis followed by haplotype analysis as implemented in GENEHUNTER (Vs. 2.1; ref. 14). To assess significance of the TDT results for each marker, we did permutation tests using the same genotype data described previously ${ }^{15,16}$. In $10^{5}$ permutations of the entire data set of 28 analyzed markers for DLG5, we observed a single $\chi^{2}$ value greater than $9.914,635$ times (empirical $P=0.046$ ), and 874 simulations had two markers with a $\chi^{2}$ value greater than 14.5 (empirical $P=0.0087$ ). We calculated pairwise LD between each marker pair and between haplotype blocks as described ${ }^{15,16}$. For case-control analysis, we calculated $\chi^{2}$ values using Fisher's exact test; we calculated genotype-based ORs using Fisher's contingency tables and tested association similarly. We calculated combined $P$ values for determining the overall significance of the observed independent association findings as outlined ${ }^{17}$.

Exon 1 identification. Because a BLAST analysis of the sequence from exon 1 as described ${ }^{10}$ showed this sequence to be derived from human mitochondrial DNA, we concluded that this sequence probably arose as an artefact of RACE amplification. We used sequence from exon 2 instead to identify expressed-sequence tags from porcine and bovine genomes containing unique $5^{\prime}$ sequences (EMBL IDs BI402246, BM484383 and BI847653). These have high similarity and could be identified within the human contig containing DLG5. This new exon of at least 300 nucleotides in the $5^{\prime}$ untranslated region is located $\sim 57 \mathrm{~kb}$ upstream of exon 2 of DLG5.

URLs. The Marshfield database is available at http://research.marshfieldclinic. org/genetics. The Genethon database is available at http://www.genethon.fr. The National Center for Biotechnology Information's SNP database is available at http://www.ncbi.nlm.nih.gov/SNP. The SNP Consortium website is available at http:///snp.cshl.org. The National Genome Research Network is available at http://www.ngfn.de.

Note: Supplementary information is available on the Nature Genetics website.

\section{ACKNOWLEDGMENTS}

We thank all cooperating clinical centers, clinicians, families and individuals with IBD and the German Crohn's and Colitis Foundation (DCCV) for their participation; J. Papp for microsatellite genotyping; A. Andersson, B. Petersen, A. Dellsén, T. Engler, M. van Giezen, Å. Jägervall, T. Kim, N. Tepe and T. Wesse for technical assistance; M. Will and T. Lu for bioinformatics support;

F. Friedrichs for assistance in statistical analysis; O. Bengtsson and

K. Forsman-Semb for discussions; and M. J. Daly for the Haploview software. This study was supported by the $5^{\text {th }}$ Framework Program of the European Commission and the Federal Ministry of Science and Education through the National Genome Research Network and the Competence Network

"Inflammatory Bowel Disease", and by a coordinated research group of the German Research Foundation (DFGFOR423).

\section{COMPETING INTERESTS STATEMENT}

The authors declare competing financial interests (see the Nature Genetics website for details).

Received 4 February; accepted 12 March 2004

Published online at http://www.nature.com/naturegenetics/

1. Hugot, J.P. et al. Association of NOD2 leucine-rich repeat variants with susceptibility to Crohn's disease. Nature 411, 599-603 (2001).

2. Ogura,Y. et al. A frameshift mutation in NOD2 associated with susceptibility to Crohn's disease. Nature 411, 603-606 (2001).

3. Hampe, J. et al. Association between insertion mutation in NOD2 gene and Crohn's disease in German and British populations. Lancet 357, 1925-1928 (2001).

4. Bonen, D.K. \& Cho, J.H. The genetics of inflammatory bowel disease. Gastroenterology 124, 521-536 (2003).

5. Hampe, J. et al. A genomewide analysis provides evidence for novel linkages in inflammatory bowel disease in a large European cohort. Am. J. Hum. Genet. 64 808-816 (1999).

6. Lu, G. et al. Inflammatory modulation of calcium-activated potassium channels in canine colonic circular smooth muscle cells. Gastroenterology 116, 884-892 (1999).

7. Nakamura, H. et al. Identification of a novel human homolog of the Drosophila dlg, P$\mathrm{dlg}$, specifically expressed in the gland tissues and interacting with p55. FEBS Lett. 433, 63-67 (1998).

8. Humbert, P., Russell, S. \& Richardson, H. Dlg, Scribble and Lgl in cell polarity, cell proliferation and cancer. Bioessays 25, 542-553 (2003).

9. Wakabayashi, M. et al. Interaction of Ip-dlg/KIAA0583, a membrane-associated guanylate kinase family protein, with vinexin and beta-catenin at sites of cell-cell contact. J. Biol. Chem. 278, 21709-21714 (2003).

10. Shah, G. et al. The cloning, genomic organization and tissue expression profile of the human DLG5 gene. BMC Genomics 3, 6 (2002).

11. Purmonen, $\mathrm{S}$. et al. HDLG5/KIAA0583, encoding a MAGUK-family protein, is a primary progesterone target gene in breast cancer cells. Int. J. Cancer 102, 1-6 (2002).

12. Lennard-Jones, J.E. Classification of inflammatory bowel disease. Scand. J. Gastroenterol. 170 (Suppl), 2-6 (1989).

13. Truelove, S.C. \& Pena A.S. Course and prognosis of Crohn's disease. Gut 17, 192-201 (1976).

14. Markianos, K., Daly, M.J. \& Kruglyak, L. Efficient multipoint linkage analysis through reduction of inheritance space. Am. J. Hum. Genet. 68, 963-977 (2001).

15. Daly, M.J. et al. High-resolution haplotype structure in the human genome. Nat. Genet. 29, 229-232 (2001)

16. Rioux, J.D. et al. Genetic variation in the $5 q 31$ cytokine gene cluster confers susceptibility to Crohn disease. Nat. Genet. 29, 223-228 (2001).

17. Fisher R.A. Statistical Methods for Research Workers 10th edn. (Oliver and Boyd, London, 1946) 